\title{
AMENDMENTS
}

\section{Author Correction: Immune and genomic correlates of response to anti-PD-1 immunotherapy in glioblastoma}

Junfei Zhao, Andrew X. Chen, Robyn D. Gartrell, Andrew M. Silverman, Luis Aparicio, Tim Chu, Darius Bordbar, David Shan, Jorge Samanamud, Aayushi Mahajan, Ioan Filip, Rose Orenbuch, Morgan Goetz, Jonathan T. Yamaguchi, Michael Cloney, Craig Horbinski, Rimas V. Lukas, Jeffrey Raizer, Ali I. Rae DD, Jinzhou Yuan, Peter Canoll, Jeffrey N. Bruce, Yvonne M. Saenger, Peter Sims, Fabio M. Iwamoto (D), Adam M. Sonabend (D) and Raul Rabadan (D)

Correction to: Nature Medicine https://doi.org/10.1038/s41591-019-0349-y, published online 11 February 2019.

In the version of this article originally published, the graph in Extended Data Fig. $2 \mathrm{c}$ was a duplication of Extended Data Fig. $2 \mathrm{~b}$. The correct version of Extended Data Fig. $2 c$ is now available online.
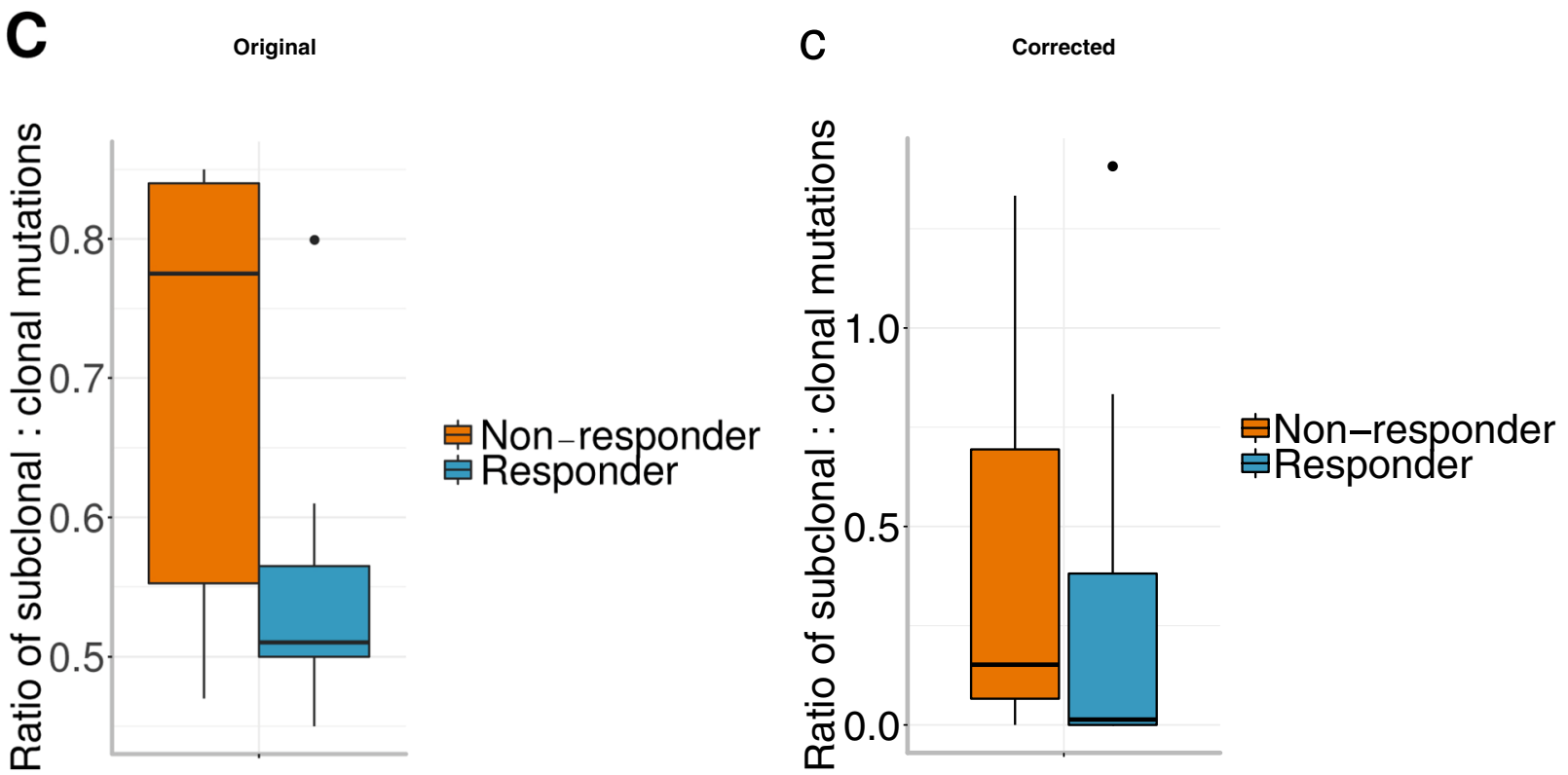

Extended Data Fig. 2c | Original and Corrected.

Published online: 17 April 2019

https://doi.org/10.1038/s41591-019-0449-8

\section{Publisher Correction: Methionine is a metabolic dependency of tumor-initiating cells}

Zhenxun Wang, Lian Yee Yip, Jia Hui Jane Lee, Zhengwei Wu, Hui Yi Chew, Pooi Kiat William Chong, Chin Chye Teo, Heather Yin-Kuan Ang, Kai Lay Esther Peh, Ju Yuan, Siming Ma, Li Shi Kimberly Choo, Nurhidayah Basri, Xia Jiang, Qiang Yu, Axel M. Hillmer (D), Wan Teck Lim, Tony Kiat Hon Lim, Angela Takano, Eng Huat Tan, Daniel Shao Weng Tan, Ying Swan Ho, Bing Lim (iD and Wai Leong Tam (D)

Correction to: Nature Medicine https://doi.org/10.1038/s41591-019-0423-5, published online 06 May 2019.

In the version of this article originally published, there is an error in Fig. 5a. Originally, 'MAT2A' appeared between 'Methionine' and 'Homocysteine.' 'MAT2A' should have been 'MTR'. The error has been corrected in the PDF and HTML versions of this article. 